\title{
Correlations between F-18 FDG PET/CT and pathological findings in soft tissue lesions
}

\author{
Hayato Nose', Hideki Otsuka², Yoichi Otomi', Kaori Terazawa', \\ Shoichiro Takao ${ }^{3}$, Seiji Iwamoto', and Masafumi Harada' \\ ${ }^{1}$ Department of Radiology, the University of Tokushima, Tokushima, Japan, ${ }^{2}$ Department of Medical \\ Imaging, the University of Tokushima, Tokushima, Japan, ${ }^{3}$ Department of Radiologic Science and Tech- \\ nology, the University of Tokushima, Tokushima, Japan
}

\begin{abstract}
Objective : To evaluate the correlations between F-18 FDG uptake imaged with PET/CT and pathological findings in soft tissue lesions. Methods : Fifty-four soft tissue lesions in 47 patients were evaluated. The correlations between the degree of FDG uptake, pathological type and grade, and MRI signal intensity and/or enhancement pattern were evaluated. Tumor FDG uptake was quantified by the maximum standardized uptake value (SUVmax). Results : Thirty-one lesions were malignant and twenty-three lesions were benign. The difference between SUVmax in the malignant and benign groups was statistically significant $(\mathbf{p}<0.001)$. Malignant myxoid lesions and well differentiated liposarcoma showed low FDG uptake. Benign neurogenic lesions showed low FDG uptake while malignant neurogenic tumors showed high FDG uptake, and the difference between SUVmax in the benign and malignant lesions was statistically significant $(p<0.001)$. In a neurofibromatosis type-1 patient who had multiple neurogenic tumors, FDG-PET/CT could distinguish malignant peripheral nerve sheath tumors from other benign lesions with similar MRI findings. Conclusions : FDG-PET/CT is useful for differentiating malignant from benign soft tissue lesions, but malignant soft tissue lesions may show various patterns on FDG-PET, and MRI may be helpful for a differential diagnosis. J. Med. Invest. 60 : 184-190, August, 2013
\end{abstract}

Keywords : F-18 FDG PET, soft tissue tumor, myxoid tumor, neurogenic tumor, liposarcoma

\section{INTRODUCTION}

F-18 fluorodeoxyglucose (FDG) positron emission tomography (PET) is useful for distinguishing between malignant and benign tumors, determining the staging of malignant disease, detecting recurrence, and evaluating the response of malignant

Received for publication January 15, 2013 ; accepted February $18,2013$.

Address correspondence and reprint requests to Hayato Nose, MD, Department of Radiology, University of Tokushima, 3-1815 Kuramoto-cho, Tokushima 770-8503, Japan and Fax : +81886-33-7174. disease to chemotherapy and radiotherapy. FDGPET has also been reported to be useful for soft tissue tumor evaluation (1-8) ; however, the numbers of reports are fewer than those for other diseases and almost all of the reports only describe sarcomas. This situation arises because soft tissue tumors are rarer than other malignant tumors, and are almost always imaged for the purpose of finding metastases and determining the staging, rather than for distinguishing between malignant and benign lesions, since it is easy to perform biopsies of these relatively more superficial soft tissue tumors.

The purpose of this study was to evaluate the 
correlations between FDG-PET/computed tomography (PET/CT) and pathological findings in soft tissue lesions. And we also evaluated findings on MRI.

\section{METHODS}

\section{Patients}

Between May 2006 and October 2011, 47 patients (25 men and 22 women; mean age, 52.1 years ; age range, $15-88$ years) with 54 soft tissue lesions were enrolled. All patients underwent MRI and FDG PET/CT before biopsy and/or surgery, and this study enrolled the lesions that were suspected to be soft tissue tumors based on these examinations. Therefore this study included both the WHO defined soft tissue tumors and the tumors and tumorlike lesions that the WHO classification dose not define as soft tissue tumor, such as lymphoma, metastasis, granulomatous lesion and hematoma.

\section{FDG-PET/CT}

All patients fasted for at least 6 hours prior to PET/CT imaging. The patients were confirmed to have a blood glucose level of $<150 \mathrm{mg} / \mathrm{dl}$ and then were intravenously injected with $3.7 \mathrm{MBq} / \mathrm{kg}$ of FDG. At $1 \mathrm{~h}$ after the FDG injection, the patients were examined using a PET/CT scanner (Aquiduo ; Toshiba Medical Systems Corporation, Tochigi, Japan). Image acquisition was performed from the top of the head to the middle of the thigh. Attenuation-corrected PET images, non-attenuation-corrected PET images, and CT images were reviewed, and the attenuation-corrected PET and CT images were coregistered using commercial software (Aquarius NET Viewer ; TeraRecon Inc., San Mateo, CA, USA). FDG uptake in the tumor was quantified by calculating the maximum standardized uptake value (SUVmax).

\section{MRI}

MRI was performed using a 1.5T scanner (Signa Excite HD ; GE Healthcare, Buckinghamshire, UK). Depending upon the location of the tumor, imaging was performed using an 8-channel cardiac coil, a single- or double-channel GP flex coil, or a single-channel knee coil. All patients underwent T1weighted (T1W), T2-weighted (T2W), and contrastenhanced T1-weighted (Gd-T1W) imaging with or without fat saturation. For Gd-T1W imaging, a standard dose $(0.1 \mathrm{mmol} / \mathrm{kg})$ of gadopentetate dimeglumine contrast agent was used. The acquisition parameters such as TR/TE, matrix size, and FOV were different for each case depending on the site of the tumor.

The correlations between the degree of FDG uptake, pathological type and grade, and MRI signal intensity and/or enhancement pattern were evaluated.

\section{Statistical Analysis}

The statistical significance of differences in SUVmax, age, blood glucose level, weight, and size between malignant and benign lesions was examined using Fisher's exact test, and p values less than 0.05 were considered to be statistically significant. The optimum cutoff value of SUVmax for the differentiation of malignancy was defined as the point on the ROC curve with the minimum distance from the $100 \%$ true-positive and $0 \%$ false-positive rate. The sensitivity, specificity, and diagnostic accuracy of FDG-PET/CT were calculated using the cutoff SUVmax value and the final diagnosis.

\section{RESULTS}

Among the 54 tumors, 31 were malignant and 23 were benign (Table 1). The SUVmax values in the malignant tumors had a range of 0.6-14.0 (mean : 5.9), while those in the benign lesions had a range of 1.2-6.2 (mean : 3.5). The difference between the SUVmax values in the malignant and benign groups was statistically significant $(\mathrm{p}<0.001)$ (Figs. 1$)$. The SUVmax had $64.5 \%$ sensitivity, $82.6 \%$ specificity, and $73.5 \%$ accuracy with a cutoff value of 4.5 . Parameters such as age, blood glucose level, weight, and size did not differ significantly between the malignant and benign groups (Table 2).

The malignant tumors showing low accumulation for SUVmax $<6.2$, which was the highest SUVmax on benign lesions, included 8 myxoid tumors, 3 well differentiated liposarcoma, 2 malignant peripheral nerve sheath tumor (MPNST), 1 synovical sarcoma and 1 metastasis of thymic squamous cell carcinoma. We investigated myxoid tumors and well differentiated liposarcoma which there were some number of cases and all cases showed low accumulation. Malignant myxoid soft tissue tumors comprised 8 lesions and showed an SUVmax range of 2-4.7 (mean : 3.1), and 3 well differentiated liposarcoma showed an SUVmax range of 0.6-3.2 (mean : 2.1). The other malignant tumors showed an SUVmax range of 3-14 (mean : 7.5). The difference between the SUVmax in well differentiated liposarcoma and 
Table 1. Summary of the histological data. MPNST means malignant peripheral nerve sheath tumor, $\mathrm{MFH}$ means malignant fibrous histiocytoma and DLBCL means Diffuse large B-cell lymphoma.

\begin{tabular}{|l|c|l|c|}
\hline \multicolumn{1}{|c|}{ Malignant } & $\mathrm{n}$ & \multicolumn{1}{|c|}{ Benign } & $\mathrm{n}$ \\
\hline MPNST & 10 & Schwannoma & 10 \\
Liposarcoma & 7 & Desmoid & 3 \\
Myxoid (3) & & Neurofibroma & 2 \\
Well differentiated (3) & & Granuloma & 2 \\
Pleomorphic (1) & & Hematoma & 2 \\
Myxofibrosarcoma & 3 & Osteolipoma & 1 \\
Myxoid chondrosarcoma & 2 & Hemangioma & 1 \\
MFH & 2 & Myositis ossificans & 1 \\
Synovial sarcoma & 1 & Xanthogranulomatous & 1 \\
Sarcoma & 1 & lesion & \\
Atypical fibroxathoma & 1 & & \\
DLBCL & 1 & & \\
Ewing sarcoma & 1 & & \\
Malignant & 1 & & \\
$\quad$ epithelioid cell tumor & & & \\
Metastasis & 1 & & \\
of thymic carcinoma & & & \\
\hline
\end{tabular}

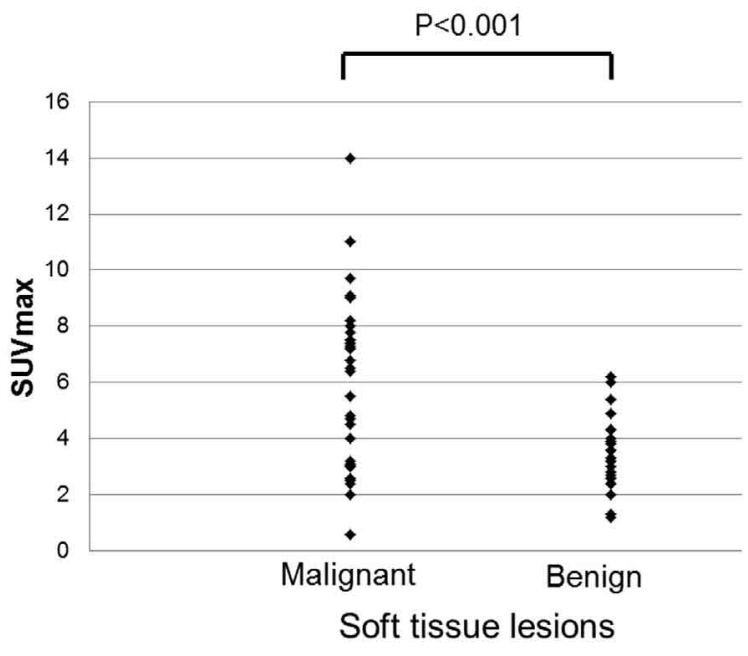

Fig. 1. SUVmax values of soft tissue lesions.

Table 2. Patient characteristics for malignant and benign lesions.

The numbers in parentheses represent the mean values.

\begin{tabular}{|c|c|c|c|}
\hline Number & Malignant & Benign & p-value \\
\hline Age & $\begin{array}{c}15-88 \\
(52.1)\end{array}$ & $\begin{array}{c}21-85 \\
(52.1)\end{array}$ & 0.990 \\
\hline Weight (kg) & $\begin{array}{c}41-92 \\
(61.8)\end{array}$ & $\begin{array}{c}44-107 \\
(61.9)\end{array}$ & 0.966 \\
\hline Blood glucose & $\begin{array}{c}77-143 \\
(95.2)\end{array}$ & $\begin{array}{c}77-113 \\
(90.8)\end{array}$ & 0.521 \\
\hline level (mg/dl) & $\begin{array}{c}23-240 \\
(77.2)\end{array}$ & $\begin{array}{c}16-225 \\
(72.3)\end{array}$ & 0.574 \\
\hline Size (mm) & $\begin{array}{c}0.6-14.0 \\
(5.9)\end{array}$ & $\begin{array}{c}1.2-6.2 \\
(3.5)\end{array}$ & $<0.001$ \\
\hline
\end{tabular}

the other malignant tumors $(\mathrm{p}<0.001)$, myxoid tumors and the other malignant tumors $(p<0.001)$, the benign lesions and the other malignant tumors $(p<0.001)$ were statistically significant. Despite confirmation of malignancy by pathology, these tumors tended to show low FDG uptake compared with the other malignant soft tissue tumors (Fig. 2-4).

Twenty-two lesions were classified as neurogenic tumors. Ten of these lesions were MPNSTs. The remaining 12 lesions were benign : schwannoma ( $\mathrm{n}=$ 10) and neurofibroma $(n=2)$. The SUVmax values of the benign neurogenic tumors had a range of 1.36 (mean : 3.2), while those of the malignant neurogenic tumors had a range of 4.5-9.7 (mean : 7.0). In the neurogenic tumors, the SUVmax values in the malignant and benign groups also differed significantly (Fig. 5). The SUVmax value had 90.0\% sensitivity, $91.7 \%$ specificity, and $98.3 \%$ accuracy with a cutoff value of 4.8. Some MPNSTs showed findings of suspected malignancy such as heterogeneous intensity, bleeding, and necrosis on MRI. However, especially in neurofibromatosis type 1 (NF-1) patients, it was difficult to identify any MPNSTs because they did not show findings of suspected malignancy on MRI, and there were numerous tumors in the whole body (Fig. 6A, 6B).

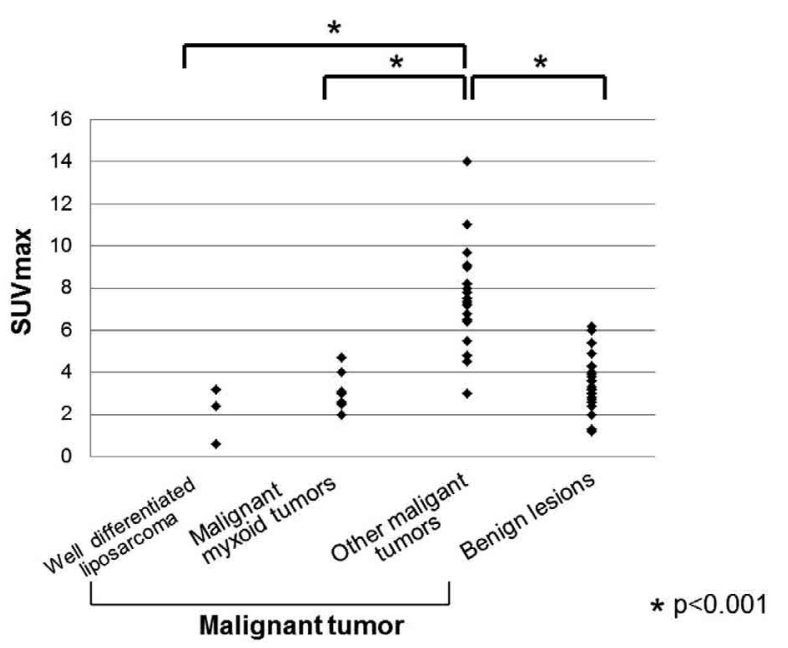

Fig. 2. Comparison of SUVmax values between malignant myxoid tumors, well differentiated liposarcoma, other malignant tumors and benign lesions. 


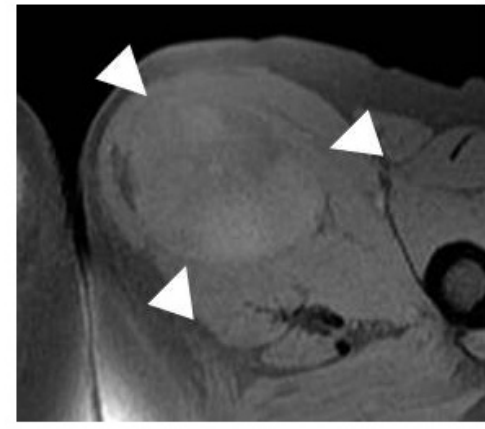

a

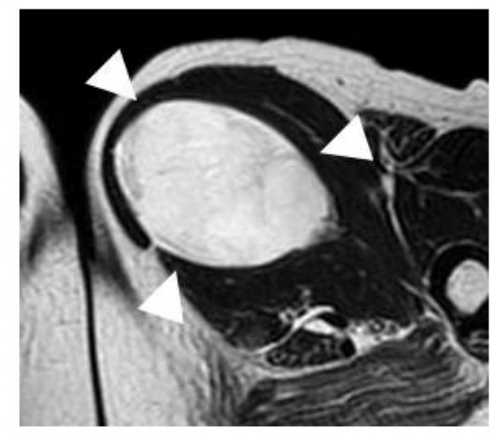

C

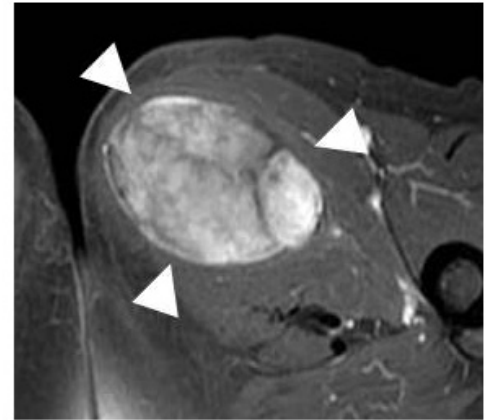

b

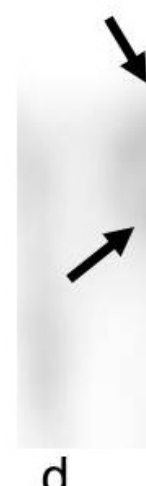

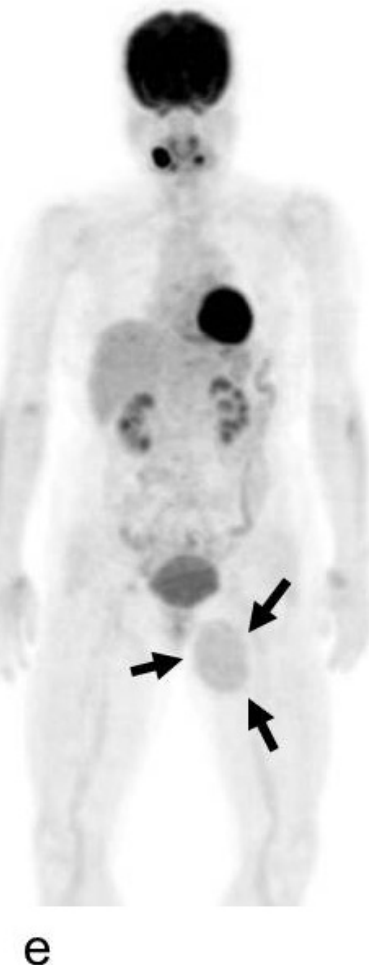

e

Fig. 3. Myxoid liposarcoma. (a-c) MR images. (d, e) FDG-PET. (a) T1W image with fat saturation. (b) Contrast-enhanced T1W image with fat saturation. (c) T2W image. The tumor showed low FDG uptake (long black arrow). The tumor showed very high intensity on T2W imaging and enhancement on Gd-T1W imaging with fat saturation (white arrow head). These findings demonstrated that the tumor included a myxoid component.

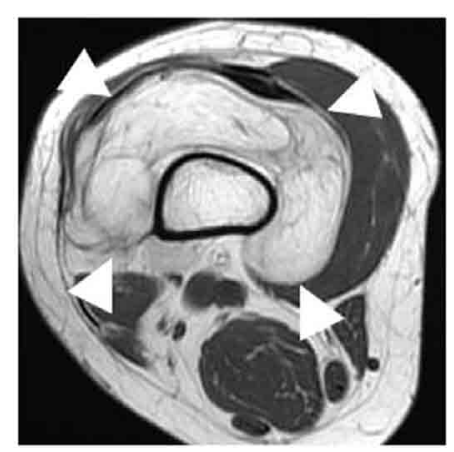

a

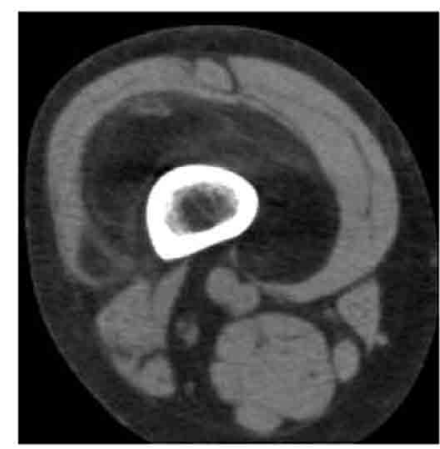

C

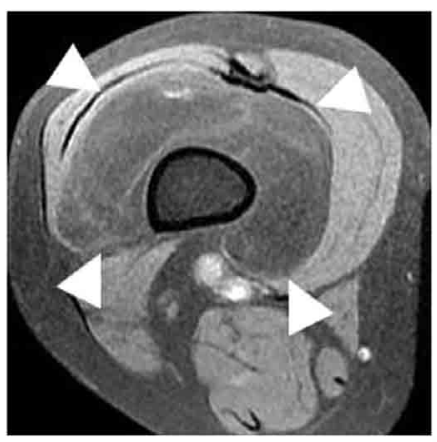

b

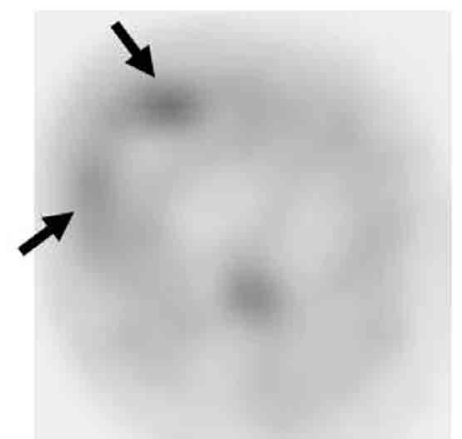

d

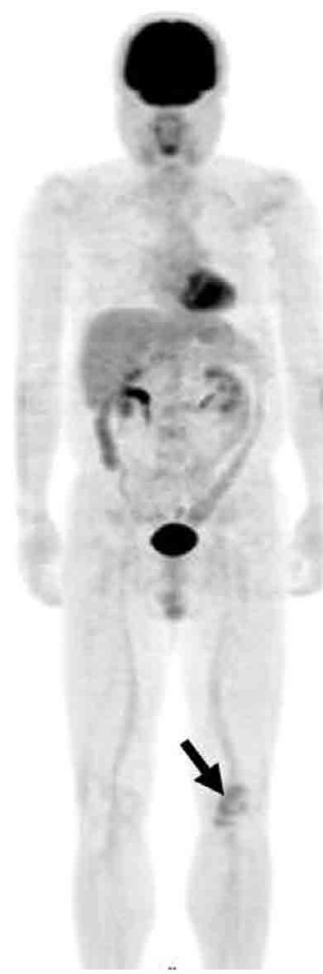

e

Fig. 4. Well differentiated liposarcoma. (a, b) MRI images. (c) CT (d, e) FDG-PET. (a) T1W image (b) T1W image with fat saturation. The tumor surrounding the thighbone consists of fat and a small amount of soft tissue lesions (white arrow head). Although the tumor shows a very low FDG uptake, soft tissue lesions of the tumor shows slightly high FDG uptake (long black arrow). 


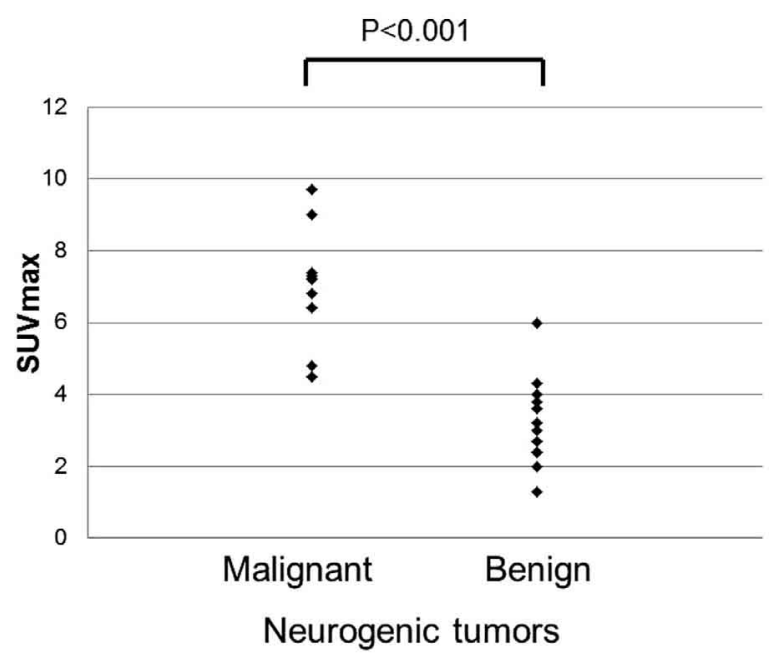

Fig. 5. SUVmax values of neurogenic tumors.
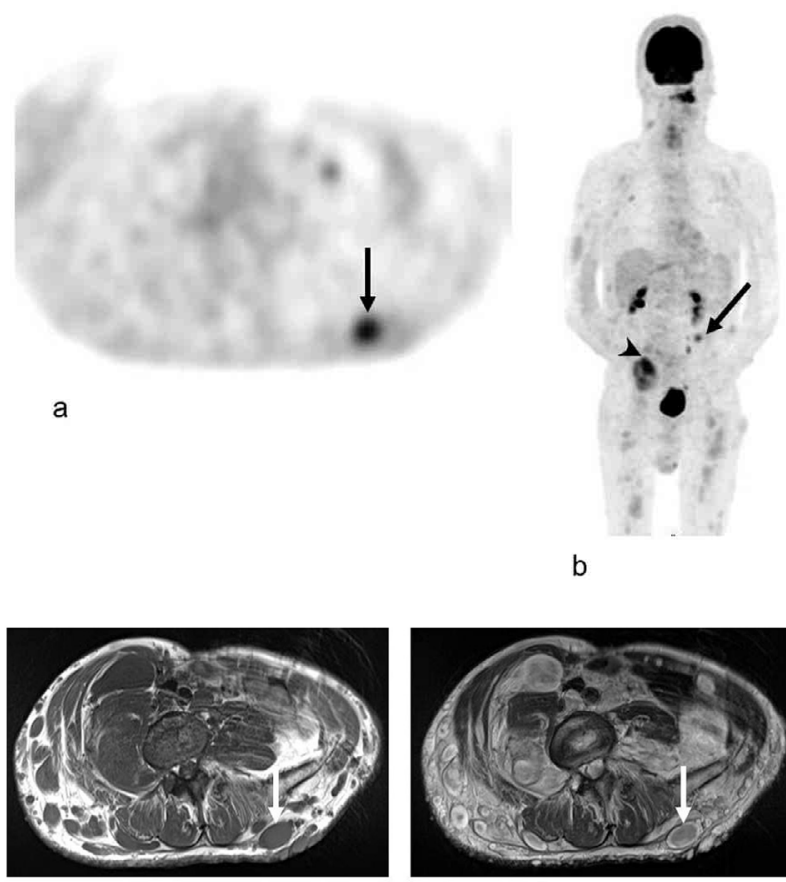

c
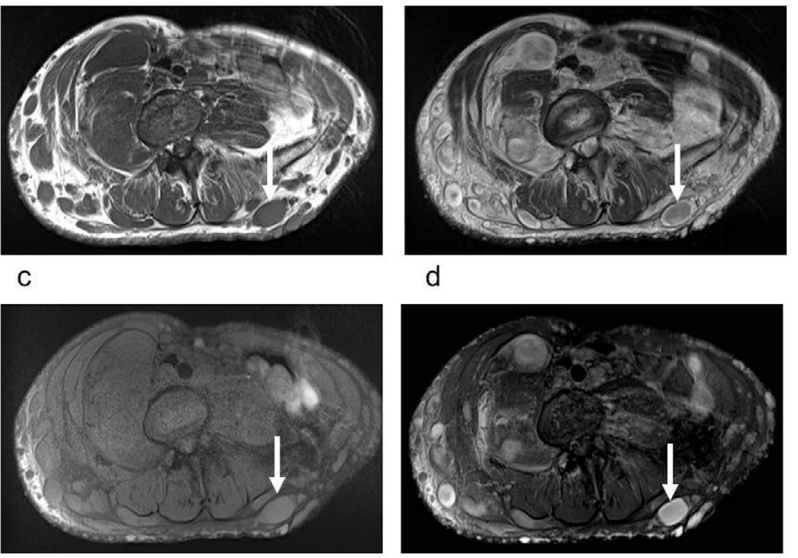

d

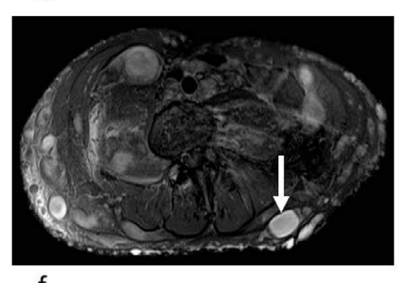

e

$f$

Fig. 6. NF-1 with low-grade MPNST. (a, b) FDG-PET. (c-f) MRI images. (c) T1W image. (d) T2W image. (e) T1W image with fat saturation. (f) Contrast-enhanced T1W with fat saturation. On FDG-PET, the tumor at the left dorsal waist showed high FDG uptake (long black arrow), indicating a low-grade MPNST. On MRI, there were numerous tumors throughout the whole body. There were no tumors showing findings of malignancy. There was a high-grade MPNST showing very high FDG uptake at the right pelvis (white arrow head).

\section{DISCUSSION}

The malignant soft tissue tumors tended to show high FDG accumulation, similar to other malignant diseases. Significant correlations were not seen between FDG accumulation and age, blood glucose level, weight, injection of FDG quantity, or tumor size.

However, the range of accumulation in malignant tumors was distributed widely, accumulation overlapped in many malignant tumors and benign lesions and there were some tumors showing false-negative findings. It was often difficult to distinguish between malignant tumors and benign lesions only by using FDG-PET.

There are some reports that accumulation in soft tissue tumors is dependent on malignancy $(3,7$, $9,10)$. Primary or secondary myxoid tumors often show low accumulation on FDG-PET, such that MRI becomes useful for diagnosis (11-15). Tateishi et al. (7) reported that tumors with a high MIB-1 index tended to have higher accumulation of FDG. They classified the tumors into three groups based on the degrees of the MIB-1 index, low (0-9), middle (1029 ), and high (>30), and reported SUVmax values of $2.6 \pm 1.6$ in the low group, $5.2 \pm 3.2$ in the middle group, and $11.1 \pm 5.0$ in the high group. However, in our study, this did not necessarily apply for myxoid tumors. The MIB-1 indices of 16 malignant lesions and four benign lesions were measured. There were four myxoid tumors measured for the MIB-1 index, and three of the four tumors had an MIB-1 index of $>30$ but showed low accumulation (SUVmax : $3.4 \pm$ 0.6). Other malignant tumors with an MIB-1 index of $>30$ showed high accumulation (SUVmax : $7.0 \pm$ 1.6). It is believed that the reason why the malignant myxoid tumors showed low accumulation is that the tumors almost completely occupied myxoid lesions and the solid malignant part was relatively small. It was difficult to distinguish between malignant tumors and benign lesions by FDG accumulation alone. However, we were able to estimate which tumors were malignant by considering the clinical and MRI findings. The finding of high intensity on T2WI and the presence of contrast enhancement was highly suggestive of malignant myxoid tumors.

Well differentiated liposarcoma showed low FDG accumulation as well. It may be that the solid malignant part of the tumor was relatively small and this type of tumor is of low malignancy. It is easy to detect liposarcoma by CT and MRI because these tumors have a large fatty component; however, it 
remains difficult to distinguish between liposarcoma and lipoma.

In these circumstances, when the soft tissue tumors show low FDG accumulation, it is important to determine whether a tumor has fatty or myxoid components by CT and MRI. If it is established that the tumor has these components, it raises the possibility that the tumor is malignant even if it displays low FDG accumulation.

There are some reports that evaluation of FDG accumulation is useful for distinguishing between malignant and benign neurogenic tumors. Although the cutoff values of SUVmax vary among such reports, many papers reported SUVmax values of 34 (10, 16-19). In our study, malignant neurogenic tumors showed high FDG uptake and benign tumors showed low FDG uptake, and the SUVmax had $90.0 \%$ sensitivity, $91.7 \%$ specificity, and $98.3 \%$ accuracy with a cutoff value of 4.8 . One benign lesion showed high accumulation (SUVmax : 6). There are many reports regarding the utility and limits of FDGPET for neurogenic tumors. It is known that benign neurogenic tumors often show high FDG accumulation based on the cell density $(10,16,17,20,21)$.

NF-1 patients have multiple neurogenic tumors throughout the whole body, and a $10 \%$ lifetime risk of MPNST development (6). Whole-body MRI is not practical for routine clinical examination except whole-body diffusion weighted MR image. But, malignant lesions, especially low-grade MPNSTs, are clinically slow-growing and frequently have an equivocal appearance to many other neurofibroma tumors on CT and MRI including DWI. Targeted needle core biopsies are often not technically feasible or are falsely negative because of the typically heterogeneous nature of these tumors, which frequently exhibit large areas of necrosis $(10,20)$. It is possible that this type of lesion can only be detected by FDG-PET/CT. These findings on PET were especially useful in the case of an NF-1 patient who had multiple neurogenic tumors compared with the MRI findings. FDG-PET was able to scan the whole body and find low-grade tumors, and is a noninvasive examination. In addition, Warbey et al. (22) reported that delayed imaging achieves greater sensitivity.

There are two limitations of our study. First, the frequency of this disease is low, thus there were few cases of each disease to evaluate. Second, when a patient was strongly suspected of having a benign lesion on imaging, they did not undergo biopsy or surgery. Consequently, they were not enrolled in the study, so the true number of benign lesions showing low FDG accumulation could be underestimated.

\section{CONCLUSIONS}

FDG-PET/CT is useful for differentiating between malignant and benign soft tissue lesions. However, malignant soft tissue tumors may show various uptake patterns in FDG-PET. Malignant myxoid soft tissue tumors and well differentiated liposarcoma in particular tended to show low FDG uptake, and these tumors were seen more frequently than other malignancies. If a soft tissue lesion shows low accumulation of FDG, CT and MRI should be examined before diagnosing the tumor as benign.

\section{FINANCIAL SUPPORT}

No potential conflict of interest relevant to this article was reported.

\section{REFERENCES}

1. Schuetze SM, Rubin BP, Vernon C, Hawkins DS, Bruckner JD, Conrad EU 3rd : Use of positron emission tomography in localized extremity soft tissue sarcoma treated with neoadjuvant chemotherapy. Cancer 103(2) : 339-348, 2005

2. Ghigi G, Micera R, Maffione AM, Castellucci P, Cammelli S, Ammendolia I, Nanni C, Barbieri E, Grassetto G, Fanti S, Rubello D : 11C-methionine vs. 18 F-FDG PET in soft tissue sarcoma patients treated with neoadjuvant therapy : preliminary results. In Vivo 23(1) : 105110, 2009

3. Yamamoto Y, Kawaguchi Y, Kawase Y, Maeda Y, Nishiyama Y : A comparative study of F-18 FDG PET and 201Tl scintigraphy for detection of primary malignant bone and soft-tissue tumors. Clin Nucl Med 36(4) : 290-294, 2011

4. Shin DS, Shon OJ, Han DS, Choi JH, Chun KA, Cho IH : The clinical efficacy of (18)F-FDG$\mathrm{PET} / \mathrm{CT}$ in benign and malignant musculoskeletal tumors. Ann Nucl Med 22(7) : 603609, 2008

5. Charest M, Hickeson M, Lisbona R, NovalesDiaz JA, Derbekyan V, Turcotte RE : FDG PET/CT imaging in primary osseous and soft tissue sarcomas : a retrospective review of 212 
cases. Eur J Nucl Med Mol Imaging 36(12) : 1944-1951, 2009

6. Iagaru A, Quon A, McDougall IR, Gambhir SS : F-18 FDG PET/CT evaluation of osseous and soft tissue sarcomas. Clin Nucl Med 31(12) : 754-760, 2006

7. Tateishi U, Yamaguchi U, Seki K, Tarauchi T, Arai Y, Hasegawa T : Glut-1 expression and enhanced glucose metabolism are associated with tumour grade in bone and soft tissue sarcomas : a prospective evaluation by $\left[{ }^{18} \mathrm{~F}\right]$ fluorodeocyglucose positron emission tomography. Eur J Nucl Med Mol Imaging 33(6) : 683-690, 2006

8. John PA, Ioannidis JL : 18F-FDG PET for the diagnosis and grading of soft-tissue sarcoma : a meta-analysis. J Nucl Med 44(5) : 717-724, 2003

9. Suzuki R, Watanabe H, Yanagawa T, Sato J, Shinozaki T, Suzuki H : PET evaluation of fatty tumors in the extremity : possibility of using the standardized uptake value (SUV) to differentiate benign tumors from liposarcoma. Ann Nucl Med 19(8) : 661-670, 2005

10. Benz MR, Czernin JC, Dry SM, Tap WD, AllenAuerbach MS, Elashoff D, Phelps ME, Weber WA, Eilber FC : Quantitative F18-fluorodeoxyglucose positron emission tomography accurately characterizes peripheral nerve sheath tumors as malignant or benign. Cancer 116(2) : 451-458, 2010

11. Conill C, Setoain X, Colomo L, Palacín A, Combalia-Aleu A, Pomés J, Marruecos J, Vargas M, Maurel J : Diagnostic efficacy of bone scintigraphy, magnetic resonance imaging, and positron emission tomography in bone metastases of myxoid liposarcoma. J Magn Reson Imaging 27 : 625-628, 2008

12. Schwab JH, Healey JH : FDG-PET lacks sufficient sensitivity to detect myxoid liposarcoma spinal metastases detected by MRI. Sarcoma 2007 ; 2007 : 36785. doi : 10.1155/2007/36785

13. Sheah K, Ouellette HA, Torriani M, Nielsen GP, Kattapuram S, Bredella MA : Metastatic myxoid liposarcomas : imaging and histopathologic findings. Skeletal Radiol 37(3) : 251258,2008

14. Schwarzbach MH, Dimitrakopoulou-Strauss
A, Mechtersheimer G, Hinz U, Willeke F, Cardona S, Attigah N, Strauss LG, Herfarth C, Lehnert T : Assessment of soft tissue lesions suspicious for liposarcoma by F18-deoxyglucose (FDG) positron emission tomography (PET). Anticancer Res 21(5) : 3609-3614, 2001

15. Enomoto $K$, Inohara $H$, Hamada $K$, Tamura $M$, Tomita Y, Kubo T, Hatazawa J : FDG PET Imaging of myxofibrosarcoma on the sphenoid sinus. Clin Nucl Med 33(6) : 421-422, 2008

16. Beaulieu S, Rubin B, Djang D, Conrad E, Turcotte E, Eary JF : Positron emission tomography of schwannomas : emphasizing its potential in preoperative planning. AJR Am J Roentgenol $182:$ :971-974, 2004

17. Ahmed AR, Watanabe H, Aoki J, Shinozaki T, Takagishi K : Schwannoma of the extremities : the role of PET in preoperative planning. Eur J Nucl Med 10 : 1541-1551, 2001

18. Otsuka H, Graham MM, Kubo A, Nishitani H : FDG-PET/CT finding of sarcomatous transformation in neurofibromatosis : a case report. Ann Nucl Med 19(1) : 55-58, 2005

19. Ferner RE, Golding JF, Smith M, Calonje E, Jan W, Sanjayanathan V, O'Doherty M : [18F] 2-fluoro-2-deoxy-D-glucose positron emission tomography (FDG PET) as a diagnostic tool for neurofibromatosis 1 (NF1) associated malignant peripheral nerve sheath tumours (MPNSTs) : a long-term clinical study. Ann Oncol 19 : 390394, 2008

20. De Waele M, Carp L, Lauwers P, Hendriks J, De Maeseneer M, Van Schil P, Blockx P: Paravertebral schwannoma with high uptake of fluorodeoxyglucose on positron emission tomography. Acta Chir Belg 105(5) : 537-538, 2005

21. Porter DE, Prasad V, Foster L, Dall GF, Birch $\mathrm{R}$, Grimer RJ : Survival in malignant peripheral nerve sheath tumours : a comparison between sporadic and neurofibromatosis type 1-associated tumours. Sarcoma $2009 ; 2009: 756395$. doi : 10.1155/2009/756395

22. Warbey VS, Ferner RE, Dunn JT, Calonje E, O'Doherty MJ : $\left[{ }^{18} \mathrm{~F}\right] \mathrm{FDG}$ PET/CT in the diagnosis of malignant peripheral nerve sheath tumours in neurofibromatosis type- 1 . Eur J Nucl Med Mol Imaging 36 : 751-757, 2009 